\title{
Inviter les patients à participer à la recherche à l'aide de la méthode photovoix
}

\author{
Candace I.J. Nykiforuk PhD
}

Citation : CMAJ 2021 July 12;193:E1050-1. doi : 10.1503/cmaj.210963-f

Voir la version anglaise de l'article ici : www.cmaj.ca/lookup/doi/10.1503/cmaj.210963; voir l'article connexe en français au www.cmaj. ca/lookup/doi/10.1503/cmaj.202537-f, et en anglais au www.cmaj.ca/lookup/doi/10.1503/cmaj.202537

$\mathbf{U}$

ne étude connexe réalisée par Campbell et ses collègues illustre adroitement la manière dont les personnes qui ont vécu ou qui vivent des problèmes de santé sont les mieux placées pour révéler et transmettre de façon évocatrice les conditions nuancées, circonstancielles et structurelles qui composent leur santé ${ }^{1}$. Ces conditions et circonstances pourraient autrement demeurer invisibles aux chercheurs, aux praticiens et aux responsables des orientations politiques, minant ultimement l'équité en matière de santé tout comme l'efficacité et l'efficience des interventions. La recherche par méthode photovoix, la méthodologie utilisée dans l'étude connexe, est une méthodologie participative faisant appel à un narratif visuel qui peut formidablement centrer la voix et le point de vue du participant dans la recherche. Un mélange de photographie et de narration, elle est décrite comme une méthode participative accessible par laquelle des patients ou une communauté sont invités à participer à un large éventail de recherche en santé2. Lorsqu'elles sont bien utilisées, les méthodes de recherche photovoix peuvent être employées pour cocréer les projets de recherche; les patients et les universitaires ou les cliniciens, chacun puisant dans ses connaissances respectives sur un problème de santé donné, travaillent de concert pour générer une question, recueillir des données et en extraire une signification pour modeler une action. Le potentiel d'enrichir la recherche en santé en offrant une avenue aux patients cochercheurs pour partager leur savoir et leurs expériences au moyen d'imageries et de récits puissants est bien réel.

La participation des patients à la recherche en santé s'est accrue en réponse aux demandes que les projets de recherche soient réalisés avec, et non sur, les patients afin de s'assurer que le travail est contextuellement, moralement et éthiquement fondé ${ }^{3}$. Idéalement, la participation des patients devrait mener à la coproduction de découvertes de recherche qui augmentent l'efficacité de l'application des connaissances et de la mise en œuvre des efforts. Pour les participants aux travaux de

\section{POINTS CLÉS}

- La recherche par méthode photovoix est une méthodologie de recherche participative qui emploie la photographie et le récit pour prioriser les expériences vécues et le point de vue des patients ou des membres de la communauté.

- L'emploi de la recherche par méthode photovoix peut rehausser la recherche faisant appel à des patients participants auprès des populations vulnérables, sur des sujets de nature délicate ou concernant la promotion de l'équité en matière de santé.

- Lorsqu'elles sont utilisées de façon inappropriée, les méthodes participatives peuvent être purement symboliques, aggravent le déséquilibre de pouvoir et perpétuent les iniquités vécues par les patients.

- Les méthodes de recherche participatives offrent un moyen d'aborder des questions de santé complexes qui mettent en jeu des déterminants de la santé non médicaux, des structures sociopolitiques et des systèmes de santé dynamiques de manière collaborative.

recherche, prendre des photos peut être plus significatif que de répondre à une enquête et moins intimidant que de participer à une entrevue ou à un groupe de discussion.

La recherche par méthode photovoix est une méthode qualitative caractérisée par une participation et une action critiques. Les personnes participent activement à la prise de photographies de l'enjeu à l'étude, un enjeu qui est important pour eux ou leur communauté, et ensuite partagent et discutent des photographies qu'ils auront sélectionnées, souvent dans le cadre d'un groupe de discussion. Par la suite, les photographies et les récits qui en résultent sont utilisés pour mobiliser les découvertes scientifiques afin d'influencer les politiques, les pratiques ou les changements communautaires auprès des responsables des orientations politiques ${ }^{4}$. La méthodologie de recherche photovoix offre une certaine autonomie aux 
participants au sein du processus de recherche; les participants choisissent ce qu'ils veulent photographier (et pourquoi), comment et quand capturer l'image et ce qui est dit de la signification de l'image lorsqu'elle est présentée aux autres. Ils ont l'occasion de gérer leurs connaissances par le processus de recherche et sa dissémination, plutôt que de les confier au chercheur pour que ce dernier les utilise à des fins d'études. L'emploi de la recherche par méthode photovoix peut être particulièrement utile pour les projets de recherche faisant appel à des communicateurs non verbaux ou des populations vulnérables, et pour les projets de recherche portant sur des sujets de nature délicate ou concernant la promotion de l'équité en matière de santé dans la conduite de l'étude ${ }^{5}$.

Les participants peuvent utiliser la photographie pour exprimer des idées ou des expériences qui, pour eux, pourraient être inconfortables ou difficiles de partager uniquement par des mots. Lorsqu'ils parlent de leurs photographies, les participants peuvent expliquer la raison pour laquelle ils ont choisi de prendre cette photo et de quelle façon les images ont une signification ou une importance pour eux, en lien avec l'enjeu à l'étude. De cette façon, les chercheurs sont invités à comprendre l'enjeu plus en profondeur, du point de vue du participant, et d'avoir une meilleure compréhension de l'expérience vécue d'une maladie, de leur capacité à accéder aux soins, sur leurs interactions avec le système de santé où des iniquités médicales et structurelles qui sous-tendent ces expériences. Les chercheurs voient l'expérience du point de vue des participants et entendent ce qu'ils disent de ces panoramas dans les mots de ces mêmes participants. Pendant les discussions de groupe, les chercheurs peuvent aussi être témoins de la manière dont les expériences des participants sont comparables ou distinctes de celles des autres participants à la conversation. Les hypothèses qui sont tenues pour acquises peuvent être explorées et défiées.

Du point de vue de la mise en œuvre scientifique, laquelle a pour but de révéler ce qui fonctionne pour chacun selon un problème de santé donné, la participation des patients à la recherche par méthode photovoix peut être formidable pour révéler les complexités de la propre santé des personnes et de leurs relations avec le système de santé de même que pour influencer la pratique ou les politiques d'intervention pour répondre à des besoins en matière de santé et de système de santé ciblé. La participation des patients à une étude par méthode photovoix devrait transcender le fait de considérer les participants comme des sources de données et plutôt les accueillir comme des cochercheurs qui aident à modeler l'enquête, contribuent aux connaissances et analysent les données de façon collaborative afin de produire de nouvelles idées à partir desquelles les décideurs poseront des actions.

Tout comme d'autres approches de recherche participative, la méthodologie de recherche photovoix incarne les principes de la justice sociale et active les projets de recherche pour prioriser et respecter l'ensemble des désirs et des contributions de connaissance des participants pour un changement communautaire. Par la recherche participative, les patients et les membres de la communauté sont bien placés pour partager leur savoir, contextualisé par leur expérience et leur point de vue, à titre de contributeurs égaux à ceux et celles offrant l'expertise scientifique et clinique. Ainsi, ils sont considérés comme experts, au même titre que les universitaires ou les chercheurs cliniciens et par leur participation aux travaux de recherche, ils deviennent des agents de changement dans leurs communautés ${ }^{6}$.

Malgré cette formidable intention réciproque, la valeur de la recherche par méthode photovoix et d'autres méthodes participatives demeure, au mieux, éclipsée par des méthodologies de recherche en santé plus conventionnelles. Dans le pire des cas, les méthodes participatives peuvent être utilisées de façon partielle ou superficielle, ne rendant que symbolique la participation du patient ou de la communauté à la recherche. La méthodologie de recherche photovoix elle-même a été sujette à de lourdes limites et à des préoccupations éthiques, y compris la priorisation des objectifs du projet de recherche sur l'action communautaire, la non-inclusion des membres de la communauté au-delà de la collecte de données, la propriété des données demeurant entre les mains des chercheurs, une fausse représentation des collaborations en recherche comme des avenues efficaces pour influencer les politiques ou le changement, une perpétuation de la rhétorique d'autonomisation et un abus de confiance entre les cochercheurs ${ }^{5}$. De plus, bien qu' " une image vaut mille mots ", la diffusion de la recherche par méthode photovoix dans le milieu universitaire est dominée par la culture écrite, reléguant souvent à un nombre minimal d'images produites par les participants à la section des résultats ou des données supplémentaires (décisions typiquement prises par le chercheur universitaire pour se conformer aux exigences de la revue scientifique et non par le cochercheur participant). Ces limites perpétuent le déséquilibre des relations de pouvoir, contredisant les fondements de Freirean ${ }^{7}$ de la recherche par méthode photovoix et nécessitant des évaluations critiques du pouvoir à travers l'ensemble du processus.

Il sera essentiel d'aborder substantiellement ces enjeux si nous voulons faire des gains durables en matière d'équité, de diversité et d'inclusion dans la façon de diriger la recherche en santé, comme recommandé par les organismes de financement fédéraux canadiens $^{8}$. Les méthodes de recherche participatives, comme la méthode photovoix, offrent un moyen d'inviter les patients et leurs communautés à aborder des questions de santé complexes qui mettent en jeu des déterminants de la santé non médicaux, des structures sociopolitiques et des systèmes de santé dynamiques de manière collaborative. Ces méthodes doivent être employées de façon rigoureuse, accompagnées d'une réflexion critique, invitant des innovations méthodologiques et des améliorations pour remédier à la sous-utilisation de la valeur des patients dans la recherche participative.

\section{Références}

1. Campbell R, Larsen M, DiGiandomenico A, et al. The challenges of managing diabetes while homeless: a qualitative study using photovoice methodology. CMAJ 2021;193:E1034-41.

2. Nykiforuk $\mathrm{CIJ}$, Vallianatos $\mathrm{H}$. Reflection/commentary on a past article: "photovoice as a method for revealing community perceptions of the built and social environment. Int J Qual Methods 2018;Aug. 28. doi: 10.1177/ 1609406918788236 
3. Bélisle-Pipon J, Rouleau G, Birko S. Early-career researchers' views on ethical dimensions of patient engagement in research. BMC Med Ethics 2018;19:21. doi: 10.1186/s12910-018-0260-y.

4. Wang C, Burris M. Photovoice: concept, methodology, and use for participatory needs assessment. Health Educ Behav 1997;24:369-87.

5. Golden T. Reframing photovoice: building on the method to develop more equitable and responsive research practices. Qual Health Res 2020;30:960-72.

6. Cargo M, Mercer SL. The value and challenges of participatory research: strengthening its practice. Annu Rev Public Health 2008;29:325-50.

7. Freire P. Pedagogy of the oppressed. New York: Routledge; 2013:131-9.

8. Tri-Agency equity, diversity and inclusion action plan for 2018-2025. Ottawa: Government of Canada; modified 2021 Apr. 14. Accessible ici : https://www. nserc-crsng.gc.ca/NSERC-CRSNG/EDI-EDI/Action-Plan_Plan-dAction_eng.asp (consulté le 7 mai 2021).
Intérêts concurrents : Aucun déclaré.

Cet article a été sollicité et il n'a pas été révisé par des pairs.

Affiliations : École de santé publique, Université de l'Alberta, Edmonton, Alb.

Propriété intellectuelle du contenu : Il s'agit d'un article en libre accès distribué conformément aux modalités de la licence Creative Commons Attributions (CC BY-NC-ND 4.0), qui permet l'utilisation, la diffusion et la reproduction dans tout médium à la condition que la publication originale soit adéquatement citée, que l'utilisation se fasse à des fins non commerciales (c.-à-d., recherche ou éducation) et qu'aucune modification ni adaptation n'y soit apportée. Voir : https://creativecommons. org/licenses/by-nc-nd/4.0/deed.fr/deed.fr.

Correspondance : Candace Nykiforuk, candace.nykiforuk@ualberta.ca 